\title{
An extension of Associative Memory approach to tracking with a drift-tube detector using timing information and its demonstration for HL-LHC ATLAS muon trigger
}

\author{
Yunjian He, Masaya Ishino, Takafumi Kodama, Masahiro Kuze, Yasuyuki Okumura, and Yohei Yamaguchi \\ on behalf of the ATLAS Collaboration
}

\begin{abstract}
High-speed online pattern recognition has been a fundamental challenge for triggering in High Energy Physics (HEP) experiments. The Associative Memory (AM) approach has been developed and used in HEP experiments for online track-finding with silicon detectors. We intend to extend the AM approach to tracking with a drift-tube detector, using the drift-time as a "new dimension" of observables in addition to spatial information. Our benchmark study demonstrates the feasibility of the extended AM concept, aiming at the online muon reconstruction with the ATLAS Monitored Drift-Tube (MDT) detector for the Phase-II Level-0 muon trigger system for High-Luminosity Large Hadron Collider. The online muon reconstruction will consist of two parts: (1) a fast track-segment finding, and (2) the following track reconstruction to estimate the momentum of the muons. It is found that timing information can be integrated into the AM approach in a natural way, and the AM approach can fit the needs for the fast track segment finding. In terms of hardware specifications expected for the Phase-II Level-0 muon trigger system, an optimal pattern training scheme is developed to prepare an effective set of AM patterns that provide high efficiency in the track-segment finding while keeping a good resolution. Based on the system-level design of electronics, an optimal algorithm chain has been developed to minimise the latency for the track segment finding. The detailed design and performance study shows that the AM approach has the capability of a high-speed and high-performance track-finding with drift-tube detectors.
\end{abstract}

\section{INTRODUCTION}

$\mathbf{M}$ AINTAINING a high-performance trigger system under the high luminosity conditions is a unique challenge towards the High-Luminosity Large Hadron Collider (HL-LHC) era [1]. High-speed pattern recognition is a key technique for performing better event selection at the trigger level, in general. The Associative Memory (AM) approach has been developed and used in the High Energy Physics (HEP) experiments for the online track-finding with silicon detectors [2]. The Associative Memory architecture is based on Content Addressable Memory (CAM) cells to identify sets of hits which are correlated on trajectories efficiently. The AM-based pattern recognition has been used to form combinations of the silicon detector hit information for track trigger applications in HEP experiments, such as CDF Silicon Vertex Tracker (SVT) [3] and ATLAS Fast TracKer (FTK) [4]. We intend an extension of the AM approach to tracking with a drift-tube detector, using the drifttime as a "new dimension" of observables in addition to spatial information. The use of timing information is found to fit the Associative Memory architecture naturally. The ATLAS Monitored Drift-Tube (MDT) system [5] provides precise measurements of muon trajectories and will be integrated into the hardware trigger system upgraded for the HL-LHC (the PhaseII ATLAS Level-0 trigger system) [6]. We have demonstrated our idea to extend the AM approach in the muon reconstruction in the Phase-II ATLAS Level-0 trigger system. The precise online reconstruction will be seeded by the fast muon trigger outputs from the dedicated trigger chambers with fast-response: Resistive Plate Chambers (RPCs) in the barrel region; Thin Gap Chambers (TGCs) in the endcap region. It consists of a straight-track finding within each detector station out of three stations ("segment finding") and a muon track reconstruction by combining the segments among the stations to evaluate the momentum of the muons.

\section{AM APPROACH WITH DRIFT-TUBE DETECTORS}

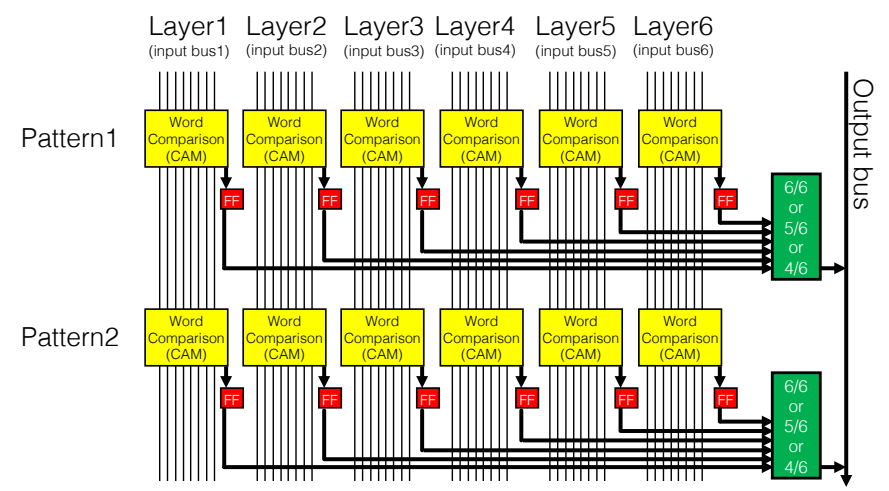

Fig. 1. A block diagram of an AM architecture. The CAM cells are shown as yellow boxes associated with flip-flops (red boxes). Green boxes indicate the word coincidence logic.

A block diagram of the AM architecture is shown in Figure 1. As an example, an architecture of two AM patterns that are based on six detector layers is illustrated. A pattern is composed of six hit-words, corresponding to the six detector layers, and the pre-calculated combinations of the hit-words are stored in the array of CAM cells. For the pattern-finding operation, an incoming hit-word from a given detector layer 
in a collision event is fed into the corresponding input databus of the Associative Memory. The incoming hit-words are compared by all the CAM cells on the data-bus simultaneously, and the flip-flop for the matching CAM cells will be latched and remain asserted until all hits from the event are received. The word coincidence logic will follow the CAM hit-matching stage to identify patterns that satisfy a programmable threshold (such as "six-out-of-six" coincidence) in the event, which are considered to be "found" patterns. A loosened requirement, such as a five-out-of-six coincidence condition, mitigates a potential hit-level inefficiency.

The patterns are pre-calculated with a large number of simulated samples ("training"), and the prepared set of patterns ("pattern-bank") is stored in the Associative Memory device. In traditional AM applications of SVT and FTK, the hit-word represents spatial points given by the binary hit channel of silicon sensors. We realise the extension to the drift-tube by expanding the hit-word representation based on the channel by adding the drift-time and left-or-right solutions. Drift-radius values which correspond to the drift-time are used in the representation. As the left-or-right solutions per hit are not known a priori, both possible solutions will be fed into the AM so that a pattern that has a correct combination of left-orright solutions will be found as the result of pattern matching. The pattern is defined as a combination of the hit-words of six layers for this application. An optimal precision of the drift-radius representation in the hit-word will be chosen in a trade-off between the resolution and pattern-bank size.

The training scheme has been optimised for the application of the drift-tube detectors, for the first time. We successfully eliminate the effects of multiple-scatterings in the training by using ideal hit information. This idea is essential to prepare a good representative set of patterns by avoiding storing patterns which are hardly matched. To maximise the pattern-finding performance for a given size of the pattern-bank, our training scheme fully exploits the advanced features of the Associative Memory device. "Ternary bit", or "don't care bit (DC bit)" functionality of the CAM realises a variable precision in the drift-radius representation of patterns so that the effectiveness of the pattern-bank is maximised [4]. "Wild-card" functionality forces specific CAM cells of individual patterns to be matched during the pattern matching operation. It is used to define patterns even where we have missing detector layers along muon trajectories due to incomplete detector acceptance. The optimal training scheme provides pattern-banks that meet the specification of the Associative Memory device concerning the size of memories.

\section{DEMONSTRATION OF SYSTEM DESIGN AND OPTIMISATION}

\section{A. System design aiming at the LHC-ATLAS Phase-II Level-O muon trigger system}

Our benchmark study demonstrates the feasibility of the extended AM concept, aiming at the online muon reconstruction with the ATLAS MDT detector for the Phase-II Level0 muon trigger system [6]. The online muon reconstruction will consist of two stages: (1) fast straight track-segment finding based on the AM approach, and (2) muon track reconstruction based on the reconstructed track-segments to estimate the momentum of the muons. The Phase-II ATLAS MDT system has three stations in the barrel region and two stations in the endcap region [7]: Barrel-Inner, Barrel-Middle, and Barrel-Outer stations in the barrel and Endcap-Middle and Endcap-Outer stations in the endcap. Straight track-segments are reconstructed in the individual five MDT stations, while other technologies will provide track-segments in the EndcapInner station. Each MDT station consists of six drift-tube layers except for Barrel-Inner station with eight layers. The diameter of the MDT tube is $3 \mathrm{~cm}$ in the current ATLAS MDT system, while small radius MDT chambers with a diameter of $1.5 \mathrm{~cm}$ will replace a part of the chambers in the Barrel-Inner station for the Phase-II system. Despite the variety of different detector configurations, our demonstration study assumes six-layers and $3 \mathrm{~cm}$ diameter in all detector stations. Angle and position correlation of the straight track-segments among stations are used in the momentum reconstruction stage.

We consider a hybrid system design using FPGA, Associative Memory devices (AMchips), and external memories, as shown in Figure 2 for this application. It shares hardware designs with Hardware-based Tracking for the Trigger (HTT) [6], which is an AM-based online silicon tracker system for the Phase-II LHC-ATLAS trigger. The hardware specification of the AMchip and the Pattern Recognition Mezzanine (PRM) developed for the HTT system is used in the design study as a benchmark. The AMchips on the PRM are the primary engines for the straight track-segment finding. The FPGA on the PRM is interfaced to optical links to receive the input hit data from detectors and to send the reconstructed straight tracksegments to another FPGA that is responsible for the muon track reconstruction. The FPGA on the PRM is interfaced to the AMchips with high-bandwidth parallel signal buses to minimise the latency.

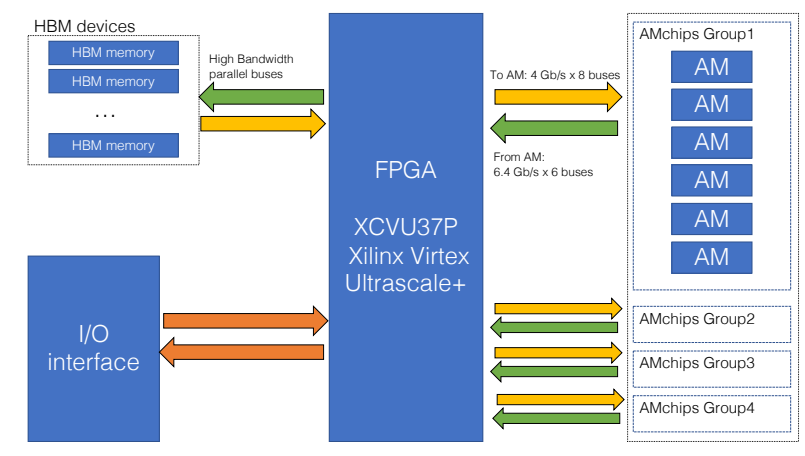

Fig. 2. A block diagram of the architecture to conduct pattern-finding of the muon segments with Associative Memory device. It consists of dedicated Associative Memory ASIC devices (AMchips), FPGA, High Bandwidth Memory (HBM) devices, and I/O interface. The hardware specification of the Pattern Recognition Mezzanine of the Hardware-based Tracking for the Trigger [6] is shared in the design.

\section{B. Optimisation of AM-based fast track-segment finding}

An optimal scheme for applying the AM approach to the tracking with the drift-tube detectors has been developed. Con- 
cerning the AMchip specification for the Phase-II HTT system, four bits for drift-radius representation with one additional bit for the left-or-right is found to be a natural solution, which corresponds to a fine granularity of $1 \mathrm{~mm}$. The DC bit provides the application of the variable resolution of the drift-radius representation, which is configurable for individual patterns and layers. The capability reduces the number of patterns while minimising the impact on the resolutions and the accidental matching rate due to detector noise. The $N_{\mathrm{DC}}^{\max }$ is the maximum allowed number of DC bits per straight tracksegment pattern. Various configurations of the $N_{\mathrm{DC}}^{\max }$ are tested to search for optimal configurations (see Figure 3). For a given $N_{\mathrm{DC}}^{\max }$, all the possible DC-bit assignments within the $N_{\mathrm{DC}}^{\max }$ for individual patterns are tested if the DC-bits can merge multiple patterns. In contrast, a full resolution will be kept for hits of patterns if the DC-bit treatment does not serve the reduction of the pattern-bank size. We choose the DC-bit configuration that satisfies the pattern-bank size requirement while minimising $N_{\mathrm{DC}}^{\max }$ as the optimal solution for each station in the pattern training. As the number of MDT tubes are different for individual stations, the needed patternbank sizes are different with a given DC-bit configuration for other stations, and therefore, the optimal configurations will be different for stations. The application of the DC bit realises solutions that satisfy the limitation for all the detector regions with the given fine granularity in the drift-radius representation.

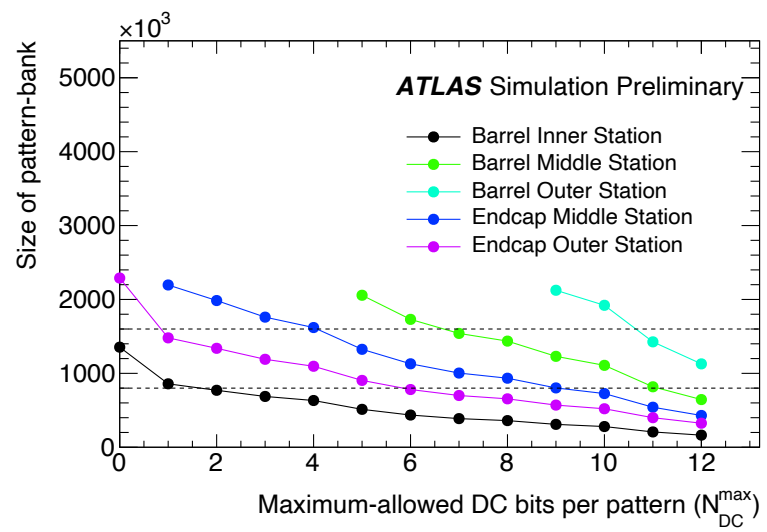

Fig. 3. The number of patterns per MDT station for various configurations of the maximum number of DC bits per pattern $\left(N_{\mathrm{DC}}^{\max }\right)$. The horizontal dashed lines correspond to the limitation of pattern size, which is $1600 \mathrm{k}$ for Barrel-Outer and Endcap-Middle stations and 800k for others. The DC-bit configuration that satisfies the pattern-bank size requirement while minimising $N_{\text {DC }}^{\max }$ is chosen as the optimal solution for each station [8].

We have developed an optimal AM-based pattern-finding operation scheme that performs hit-word coincidence with three different thresholds simultaneously; six-, five-, and four-out-ofsix. The use of loosened conditions allows a robust operation despite possible tube-hit inefficiencies due to various reasons. At the same time, a prioritised readout for the three conditions will enable us to use the best estimate without a cost of latency to readout other patterns that are supposed to have lower quality. In training, representative track-segment parameters (positions, angles, number of simulated muons represented by the individual patterns, and the standard-deviation of the position and angle estimates) are calculated for all individual patterns. They can be stored in the external memories, which will be used as look-up tables. Therefore, as soon as the segment finding by the AM completes, these parameters can be retrieved from the memories without additional latency to calculate the track-segment parameters. The FPGA sends the information of the track-segments to another FPGA which is in charge of combining the track-segment information from different detector stations and of the momentum reconstruction.

The latency for the straight track-segment finding is expected to be $477 \mathrm{~ns}$ (see Table I). The latency is estimated under the assumption that the system clock runs at $200 \mathrm{MHz}$ and $250 \mathrm{MHz}$ at the AMchips and the FPGAs, respectively. The "pre-AM" logic in the Table includes hit extraction with respect to the Region-of-Interest pointed by the RPC and TGC hit information while post-AM logic contains prioritisation logic for the three different thresholds and the extraction of the tracksegment parameters from the external memory devices.

TABLE I

EXPECTED LATENCY FOR THE STRAIGHT TRACK-SEGMENT RECONSTRUCTION.

\begin{tabular}{ll}
\hline \hline & latency [ns] \\
\hline \hline Transceiver SerDes latency + FIFO & 128 \\
Pre-AM logic & 20 \\
FIFO at FPGA + Latch at AM & 16 \\
AM segment finding & 100 \\
Pattern readout & 25 \\
Post-AM logic & 60 \\
Transceiver SerDes latency + FIFO & 128 \\
\hline Total & 477 \\
\hline \hline
\end{tabular}

\section{The optimal method of online muon reconstruction and the momentum estimation}

The estimation of transverse momentum $\left(p_{\mathrm{T}}\right)$ of muons will follow the online AM-based fast straight track-segment finding. The angle and position information in the individual stations is used to reconstruct the muon tracks and estimate the momentum of the muons.

We consider three types of observables that can be obtained by reasonably simple calculations: $\alpha, \beta$, and sagitta $(s)$ observables as proxies of $p_{\mathrm{T}}$ of muons (see Figure 4). The Muon spectrometer consists of three stations (Inner, Middle, and Outer) in the Barrel region, and the three observables can be evaluated using the straight track-segments in the three stations. They are proxies for the curvature of muon trajectories in the toroidal magnetic field and approximately proportional to the inverse of the $p_{\mathrm{T}}$ of the muons. The observable $\alpha$ is defined in the middle or outer station as the angle difference between the direction of the segments and straight trajectories pointing to the detector origin, corresponding to trajectories with infinite momentum; $\beta$ is the angular difference of the direction of segments in two different MDT stations; the sagitta is defined as the distance from the position of the segment in the middle MDT station to a straight line connecting the segment positions in the inner and the outer MDT stations. The three types of observables can be used in the online $p_{\mathrm{T}}$ estimation, and thus for the selection in triggering high- $p_{\mathrm{T}}$ 
muons. These estimates have different resolutions depending on the length of lever arms. The sagitta will provide the best resolution in the $p_{\mathrm{T}}$ estimation of the three while $\beta$ and $\alpha$ mitigate the effect of inefficiency in the straight track-segment reconstruction. Thus, if the straight track-segments are found in all three MDT stations, six observables can be available for the estimation of muon $p_{\mathrm{T}}$ : two $\alpha$ 's at the middle and outer stations, three $\beta$ 's for the possible three combinations of two stations, and the sagitta $(s)$. If more than one observable is available, the one with the best resolution will be used in the $p_{\mathrm{T}}$ decision, based on the simulation results. The variables for the endcap are defined similarly from the position and the angle of the track segments [6].

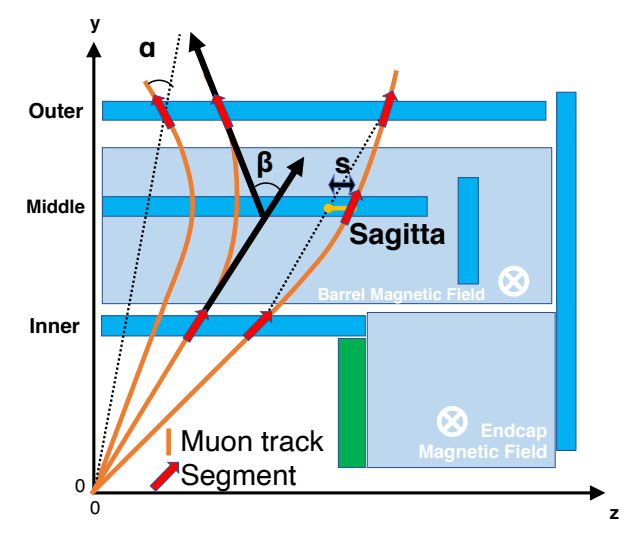

Fig. 4. The definitions of three observables $(\alpha, \beta$, sagitta $(s))$ that are used in the demonstration study of online muon reconstruction with the Associative Memory approach. They are proxies for the curvature of muon trajectories in the toroidal magnetic field and approximately proportional to the inverse of muon $p_{\mathrm{T}}[8]$.

\section{PERformance STUdy}

We have demonstrated performance of the developed methods of the AM-based straight track-segment reconstruction and online muon momentum estimation in terms of the following three points: (1) resolutions of the track-segment reconstruction, (2) efficiency of the selections with the precise momentum information given by the online, and (3) trigger rate reduction for various background conditions. The developed online muon reconstruction system is emulated by a software-based algorithm with respect to the design described in Section III. The AM-based online reconstruction simulation runs on either simulated samples or actual collision data taken by ATLAS in 2018 during the LHC Run 2 with a centre-of-mass energy of $13 \mathrm{TeV}$ and a bunch-crossing interval of $25 \mathrm{~ns}$.

We study the performance of the track-segment finding, using simulated $Z \rightarrow \mu \mu$ samples in a background environment compatible to the HL-LHC with an average number of collisions per bunch crossing, denoted as $\langle\mu\rangle$, of 200 . The track-segment reconstruction method described above with the prepared sets of the pattern-banks after the DC bit application is found to provide a good position resolution (200-370 $\mu \mathrm{m})$ and angular resolution (1.0-2.4 mrad), varying for different detector stations. The efficiency is as high as $99 \%$ in a short latency as shown in Table I, even in the background environment expected for the HL-LHC operation. Figures 5 (a) and (b) show the residual of angle and position of the reconstructed straight track-segment between the AM-based pattern finding and software-based offline tracking at the Barrel Inner station, respectively.

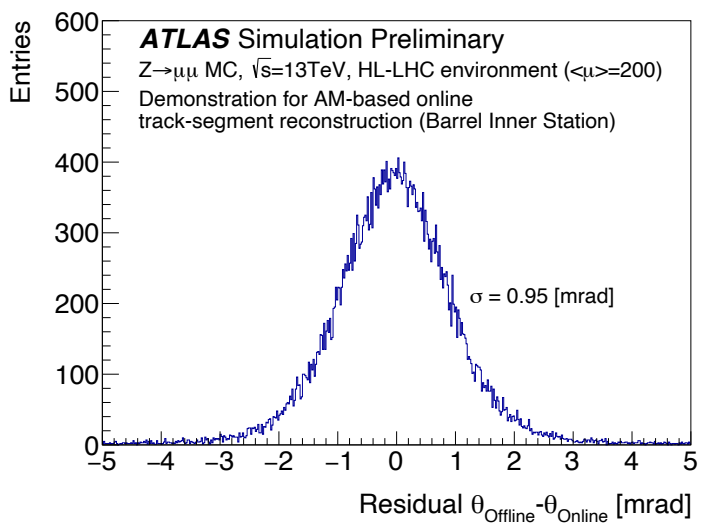

(a)

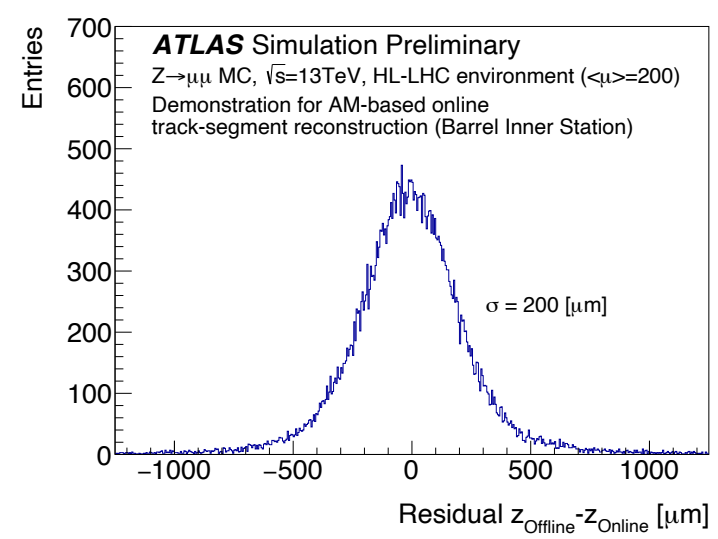

(b)

Fig. 5. Online straight track-segment reconstruction with the Associative Memory approach runs for offline-reconstructed straight track-segments at the three muon stations in the Barrel region (Barrel Inner station), and the residual distributions of (a) angle $\theta$ and (b) position $z$ of straight tracksegments between the offline and the online reconstruction are shown. The pattern-finding algorithm with the AM approach will find offline segments for given sets of MDT hits at three muon stations, and representative $\theta$ values of AM patterns $\left(\theta_{\text {online }}\right)$ and $z$ values of AM patterns $\left(z_{\text {online }}\right)$ are used as the online estimations. For the study, $Z \rightarrow \mu \mu$ processes are simulated with the background environment expected for the High-Luminosity LHC. The residual distributions correspond to the angular resolution of $\sigma=0.95 \mathrm{mrad}$ and position resolution of $\sigma=200 \mu \mathrm{m}$ in the Barrel Inner station [8].

We collect good quality offline reconstructed muons for the demonstration study. They are required to be associated with Run-2 Level-1 single muon triggers with $p_{\mathrm{T}}$ threshold at $20 \mathrm{GeV}$ (L1_MU20) [9] in the barrel region (i.e. within the pseudorapidity of $\left.\left|\eta^{\text {RoI }}\right|<1.05\right)$. The detector location found by the Level-1 trigger is called the Region-of-Interest or RoI. Matching between the offline muon and the L1_MU20 RoI requires $|\Delta \eta|<0.08$ and $|\Delta \phi|<0.18$ (difference of azimuthal angles), where $|\Delta \eta|$ and $|\Delta \phi|$ are calculated from $\eta$ and $\phi$ of the offline-reconstructed muon and the central position of the L1_MU20 RoI. The Run-2 L1_MU20 candidates are collected without selection in the higher-level trigger, exploiting a pass- 
through mode trigger seeded by the L1_MU20.

Figure 6 shows the $p_{\mathrm{T}}$ distribution of the offline muons. The AM-based online straight track-segment reconstruction is emulated at each station. The muons are classified into categories for the number of available online track-segments for the momentum determination, which determined the available observables for the momentum estimation. About $90 \%$ of the muons have online-segments reconstructed at all three stations ("3 stations") and can use the sagitta method in the online momentum determination. Muons categorised into "2 stations" and "Middle or Outer 1 station only" (about 10\%) can be reconstructed by the $\beta$ method and the $\alpha$ method, respectively. These methods mitigate the inefficiency of the track-segment reconstruction. The remaining small fraction of muons (less than $0.15 \%$ ) fails the online momentum reconstruction due to the lack of available online track-segments, categorized into either of "Inner Only" or "0 station" in the figure. Except for the $0.15 \%$ muons that fail the online momentum reconstruction, a refined selection is applied in order to improve the selectivity on top of the Run-2 L1_MU20 trigger selection. In Figure 7 (a), the filled histogram with blue colour shows the $p_{\mathrm{T}}$ distribution of muons that satisfy the $p_{\mathrm{T}}$ threshold for $20 \mathrm{GeV}$ trigger with the refined momentum information provided by the online muon reconstruction with AM approach, denoted as "Refined L1_MU20". In contrast, the total offline muons matched to the Run-2 Level-1 RoI of L1_MU20 before the application of the refined selection are illustrated with the open histogram. Figure 7 (b) shows the relative efficiency of the refined transverse momentum selection provided by the online reconstruction using the AM approach with reference to the offline matched to the Run-2 Level-1 RoI of L1_MU20. The online reconstruction with the AM approach provides additional rejection capability for muons with $p_{\mathrm{T}}<20 \mathrm{GeV}$ while it keeps high efficiency for muons with $p_{\mathrm{T}}>20 \mathrm{GeV}$. A plateau efficiency is found to be as high as $99.5 \%$. The efficiency rises for muons below $6 \mathrm{GeV}$ as the system-design and AM pattern bank is optimised for muon with $p_{\mathrm{T}}>6 \mathrm{GeV}$ in this demonstration study. The offline $p_{\mathrm{T}}$ measured by the muon spectrometer with a constant offset correction corresponding to the energy deposit in the materials in front of the muon spectrometer $(2.8 \mathrm{GeV})$ is taken as the $x$-axis in the figures.

Further, the expected trigger rate reduction relative to the Run-2 L1_MU20 has been evaluated in the various luminosity conditions using actual collision data taken in 2018 (see Figure 8). The Run-2 L1_MU20 candidates are collected by the pass-through mode of the High Level Trigger seeded by the L1_MU20 in 2018 data within $\left|\eta^{\text {RoI }}\right|<1.05$ without bias owing to the high-level software-based trigger algorithm. Figure 8 shows the relative trigger rate of the Refined L1_MU20 with respect to the Run-2 L1_MU20 as a function of the average number of interactions per bunch crossing. The refined selection using muon transverse momentum provided by the online muon reconstruction with the AM approach offers 50\% trigger rate reduction while keeping high efficiency for the high- $p_{\mathrm{T}}$ muons. The performance does not depend on the pileup in the range up to $\langle\mu\rangle$ of 55 , corresponding to various instantaneous luminosities and, thus, different background

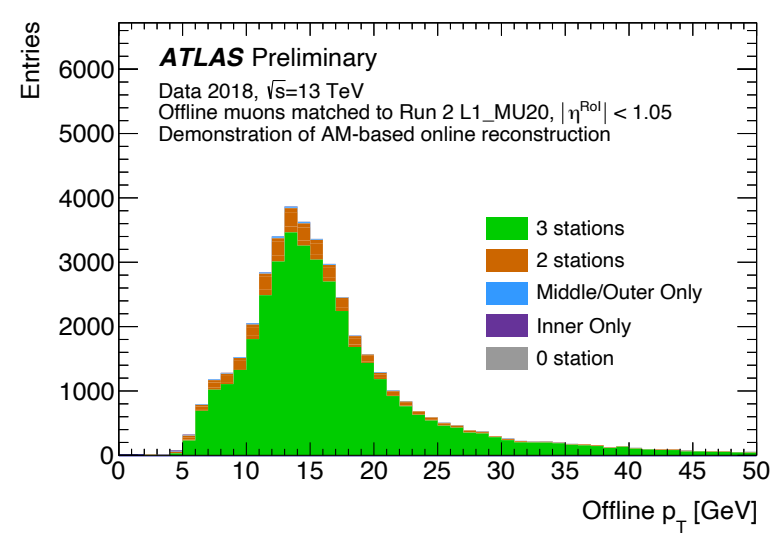

Fig. 6. The $p_{\mathrm{T}}$ distribution of offline reconstructed muons matched to a Level1 RoI of L1_MU20 within $\left|\eta^{\text {RoI }}\right|<1.05$. The muons are classified into five categories for the number of available online track-segments for the momentum determination. About $90 \%$ of the muons have online-segments reconstructed at all three stations and, therefore, the sagitta method is applicable (" 3 stations"). Furthermore, another $10 \%$ of the muons which do not have three tracksegments can be reconstructed by either of $\beta$ or $\alpha$ method if we have tracksegments available in two out of three stations or one track-segment available in either of Middle or Outer station (" 2 stations" and "Middle/Outer Only", respectively). The remaining small fraction of muons (less than $0.15 \%$ ) fail the reconstruction of enough number of online track-segments to estimate the momentum ("Inner Only" and "0 station") [8].

conditions. Besides, the figure shows $60 \%$ of muons with $p_{\mathrm{T}}<20 \mathrm{GeV}$ are rejected by the refined selection given by the online muon reconstruction with the AM approach.

\section{CONCLUSION}

We intend to extend the AM approach to tracking with a drift-tube detector, using the drift-time as a "new dimension" of observables in addition to spatial information. Our study shows that the use of timing information of drift-tube detectors naturally fits the Associative Memory architecture. We have successfully demonstrated the realistic system-level optimisation, aiming at the online muon reconstruction with the ATLAS Monitored Drift-Tube detector for the Phase-II Level-0 muon trigger system. Exploiting features of AMchip devices, a system-level design of the fast online muon reconstruction system has been developed with respect to the given set of hardware specifications and the limitation. The system will consist of the following two stages; (1) straight track-segment reconstruction within each detector station out of three stations ("segment finding"), and (2) muon momentum evaluation by combining the segments among the stations. An optimal solution of system-level design is found and the methodology of the system optimisation has been established. The performance has been checked by using the hardware simulation running on both the simulated samples and the actual collision data taken in 2018 during the LHC-ATLAS Run-2 operation. The refined selectivity provided by the online muon reconstruction based on the AM approach saves 50\% of the bandwidth of data recording while sustaining high efficiency for high- $p_{\mathrm{T}}$ muons with a plateau efficiency of $99.5 \%$, which demonstrates the feasibility of the actual application based on our new ideas. 


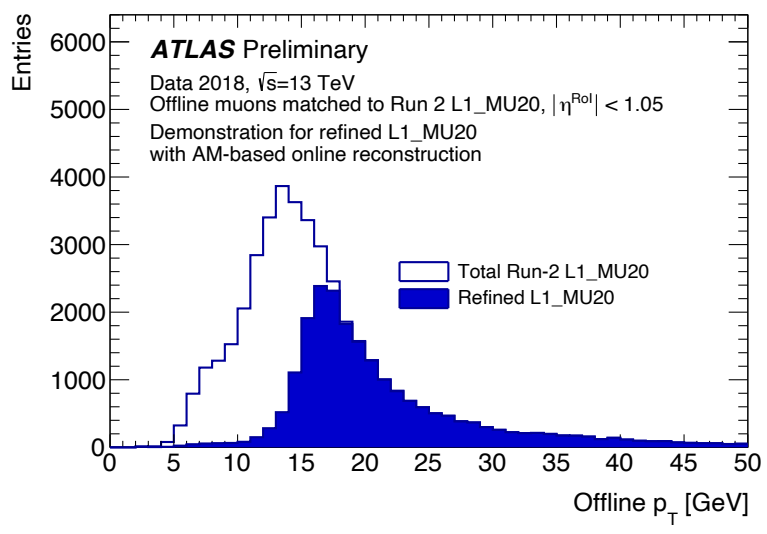

(a)

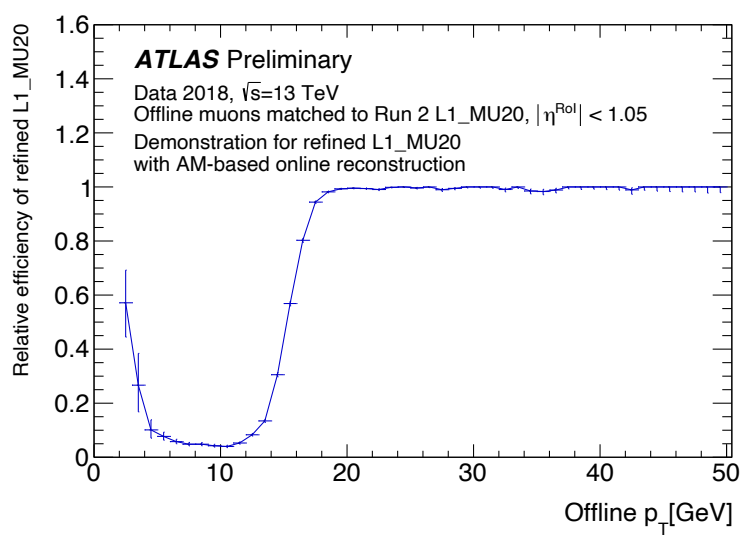

(b)

Fig. 7. (a) The $p_{\mathrm{T}}$ distribution of offline reconstructed muons matched to a Level-1 RoI of L1_MU20. The filled histogram with blue colour shows the $p_{\mathrm{T}}$ distribution of muons that satisfy the $p_{\mathrm{T}}$ threshold for $20 \mathrm{GeV}$ trigger with the refined momentum information provided by the online muon reconstruction with AM approach, denoted as "Refined L1_MU20" in the figure. (b) The relative efficiency of the refined $p_{\mathrm{T}}$ selection provided by the online reconstruction using AM approach with reference to the offline muons matched to a Level-1 RoI of L1_MU20. The online reconstruction with the AM approach provides additional rejection capability for muons with $p_{\mathrm{T}}<20 \mathrm{GeV}$ while it keeps high efficiency for high- $p_{\mathrm{T}}$ muons with a plateau efficiency of $99.5 \%$. The efficiency rises for muons below $6 \mathrm{GeV}$ as the system-design and AM pattern list is optimised for muon with $p_{\mathrm{T}}>6 \mathrm{GeV}$ in this demonstration study. The offline $p_{\mathrm{T}}$ measured by the muon spectrometer with a constant offset correction corresponding to the energy deposit in the materials in front of the muon spectrometer $(2.8 \mathrm{GeV})$ is taken as the $x$-axis in the figure [8].

\section{ACKNOWLEDGEMENT}

This work was partially supported by JSPS KAKENHI Grant Number $17 \mathrm{H} 02880$.

\section{REFERENCES}

[1] Apollinari, G. et al., CERN Yellow Rep. Monogr. 4 (2017), CERN-2017007-M.

[2] F. Morsani et al., "The AMchip: A VLSI associative memory for track finding", Nucl. Instrum. Methods Phys. Res. A315, 446 (1992).

[3] S. Belforte et al., "The CDF trigger silicon vertex tracker (SVT)", IEEE Trans. Nucl. Sci. 42, 860 (1995); J. Adelman et al., "The Silicon Vertex Trigger upgrade at CDF", Nucl. Instrum. Methods Phys. Res. A572, 361 (2007).

[4] ATLAS Collaboration, "Fast TracKer (FTK) Technical Design Report", CERN-LHCC-2013-007, ATLAS-TDR-021.

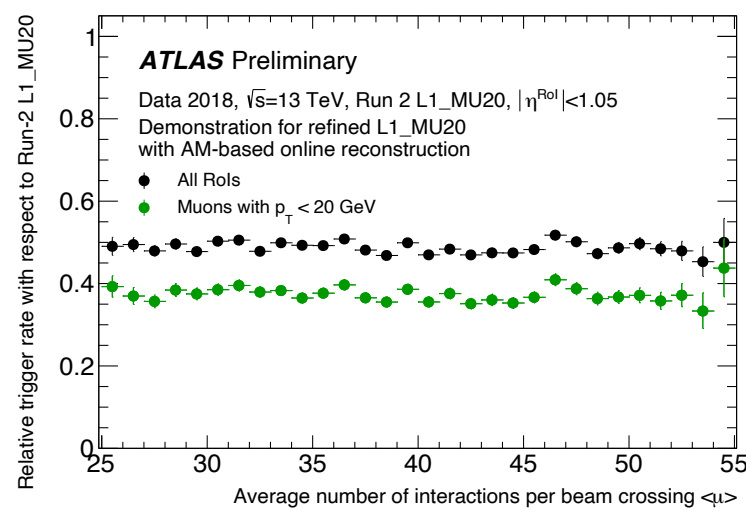

Fig. 8. The relative trigger rate of the Refined L1_MU20 with respect to the Run-2 L1_MU20 (black points) as a function of the average number of interactions per bunch crossing. Additional 50\% rate reduction can be provided by the refined selection, and the performance does not depend on the pileup in the range up to $\langle\mu\rangle$ of 55 . In addition, the figure shows $60 \%$ of muons with $p_{\mathrm{T}}<20 \mathrm{GeV}$ are rejected by the refined selection given by the online muon reconstruction with the AM approach (green points) [8].

[5] ATLAS Collaboration, "The ATLAS Experiment at the CERN Large Hadron Collider", JINST 3 (2008) S08003.

[6] ATLAS Collaboration, "Technical Design Report for the Phase-II Upgrade of the ATLAS TDAQ System", CERN-LHCC-2017-020, ATLAS-TDR029.

[7] ATLAS Collaboration, "Technical Design Report for the Phase-II Upgrade of the ATLAS Muon Spectrometer", CERN-LHCC-2017-017, ATLASTDR-026.

[8] ATLAS Collaboration, "L0MuonTriggerPublicResults", https: //twiki.cern.ch/twiki/bin/view/AtlasPublic/L0MuonTriggerPublicResults (accessed: 2020-12-10)

[9] ATLAS Collaboration, "Performance of the ATLAS muon triggers in Run 2", JINST 15 (2020) P09015. 